\title{
Application of Data Warehouse and Data Mining Technology in Power System
}

\author{
Haiyan Hao \\ Hainan College of Software Technology, 571400, Shina
}

Keywords: Data Warehouse, Data Mining Technology, Power System, Research.

\begin{abstract}
Data warehouse and data mining technology are two very important technologies at present. in the information age, data show a large number and rapid growth. how to deal with the current changing development environment has become the main content of public concern, data processing has become extremely important in the era of big data. this paper discusses the concept of data warehouse and data mining technology, and discusses the application of data warehouse and data mining technology in power system.
\end{abstract}

\section{Introduction}

With the development of the economy towards integration, the market economy environment becomes more complex, enterprises should pay attention to the market development state in this period, but also should understand the market demand, after considering the factors affecting the development of the enterprise in all aspects, they can make scientific and accurate prediction according to the information already available, the database shows the strong working ability in application, it can deal with a lot of data and improve the work efficiency, and it can also reduce the error of manual work. However, with the development of the network platform, the number of people using the Internet is increasing gradually, in order to increase rapidly. In this process, enterprises not only need to integrate data, but also need to collect and integrate data, make a correct judgment on the content of information in a short period of time, and the database and data are mining technology in this context.

\section{Data Warehouse and Data Mining Concepts}

\subsection{Data Warehouse}

Data warehouse, as its name implies, is a shelter for data, and it has a powerful function, which combines the elements of subject-oriented, time-varying, data integration, stability and so on organically. The database is not simply a collection of data, but also can provide a solid data base for managers to plan the development direction of the enterprise through the data processing, and the database ability is powerful, and the decision-making effect is shown in figure 1.[1].

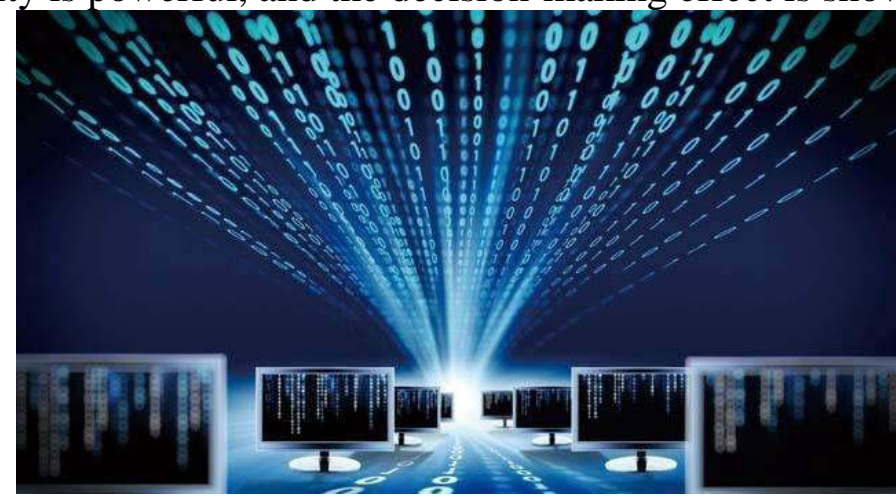

Figure 1 Data warehouse structure

On the basis of the original data collection technology, the database can integrate and classify the data stored in the database according to certain requirements, and after collecting the data, it can analyze the data synchronously, the strong data analysis ability of the database, analyze the data 
through the data relational database, understand the data composition, this process of the database will be very abstract, the topic attempt content is the data in essence, just a new way of presentation, the database integrates, processes, analyzes and so on, needs to adjust the data structure, after reorganization, the data can be standardized processing, so that the data can be collected in a short time, through collation, analysis, to obtain valuable information, to provide support for the management personnel to formulate the program, etc.[2].

Data integration performance is also a very prominent feature of the database, integration is to extract data from each subsystem, and according to a certain logical relationship of integration, after processing, decentralized data form cannot be used directly, data warehouse theme is to understand the needs of enterprise work, flexible according to the needs of the market, to improve the previous technology, under the role of data warehouse theme, the source data will be based on the decentralized database, through the form of commands, to process the repeated data in the decentralized database, and according to the logical relationship, adjust the data analysis, through calculation, add execution, delete operation, and form a unified concept definition.

The database also has a special characteristic, its data cannot be updated, the update here is different from the daily software update, after the database considers the data security problem in the work, so it is specially designed, the data stored in the database cannot be deleted, can only be queried by programming command, analysis data, collate data, because the data warehouse has this special performance, also make the data warehouse internal environment very stable[3].

The database has strong performance in application, it can guarantee the security of data in the period, and the database is more powerful in application and analysis. With the change of time, the data in the database will change to a certain extent, the historical information stored in the database will be transferred from the database to the historical data after a period of time, and the high-level data integration will continue after the conversion, and after the continuous synthesis and conversion, the frequently used data will become very active. In contrast, the data that is not often used will flow to the edge, if this situation does not change, after a period of time, the data will be transferred from the database to the relatively low price equipment, and the data analysis will be more powerful after a period of time.[4].

\subsection{Data Mining}

Data mining and data warehouse are both effective ways of data analysis in the era of big data. With the development of information technology and the optimization of science and technology, the storage capacity of data warehouse has been increasing in the same period, now it has been expanded to hundreds of $\mathrm{TB}$, and the data warehouse capacity still shows a growing trend, that is, the storage range of data warehouse will be further expanded in the future, and the data warehouse capacity will be expanded continuously, at the same time, the more powerful data analysis tool is needed to extract valuable information and support decision-making, which can facilitate the enterprise managers to process the daily work more easily, the data mining can make full use of the data mining work, and provide the decision-making data for the managers with the rapid and accurate data warehouse capacity. Knowledge Discovery in Database From here we can understand the data development stage, the integration between artificial intelligence and data mining, data mining is an important means to ensure the ability of data analysis in the process of data analysis, Knowledge Discovery in Database has more links, each link needs a specific algorithm to ensure that the data mining work can proceed smoothly, but also should be based on the current information, data preparation, mining, evaluation and other work, figure 2 is the data mining algorithm model. There are many methods for data mining, such as decision tree, fuzzy set, neural network and so on.[5]. 


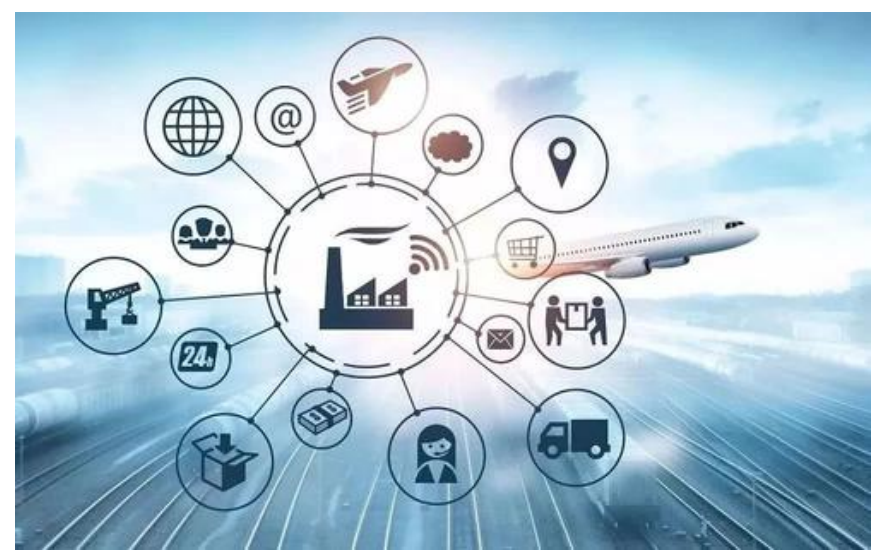

Figure 2 Big data era

\section{Application of Database and Data Mining}

With the modernization process, the electric power market environment gradually becomes standard and orderly, in this environment also needs to further construct the electric power automation and the information system, with the economic integration development, the electric power industry needs to follow the pace of the times development, to the intelligent, the automation direction development, guarantees the electric power enterprise to obtain the bigger development space through the reform in the development, and will obtain the objective economic benefit, therefore many electric power enterprises introduce the data base and the data mining technology in the work.

\subsection{Information Management}

Database and data mining technology can deal with a large amount of data in a short period of time, which can greatly improve the efficiency of electric power enterprises. In the past, the enterprise records the daily expense data, simply records the daily expense, the electric power enterprise purchases and so on, although the work volume is small, but also needs to spend a lot of money, in this process, it is also difficult to ensure the reasonableness of each work, in order to improve the efficiency, to ensure the maximum value of each fund, to use the database and data mining technology, so that the problem can be solved well. After analyzing the purchase order data and the logistics work database, the purchasing work can be optimized according to the data analysis result, so that the power enterprise needs to change the power supply mode in order to respond to the call of environmental protection.

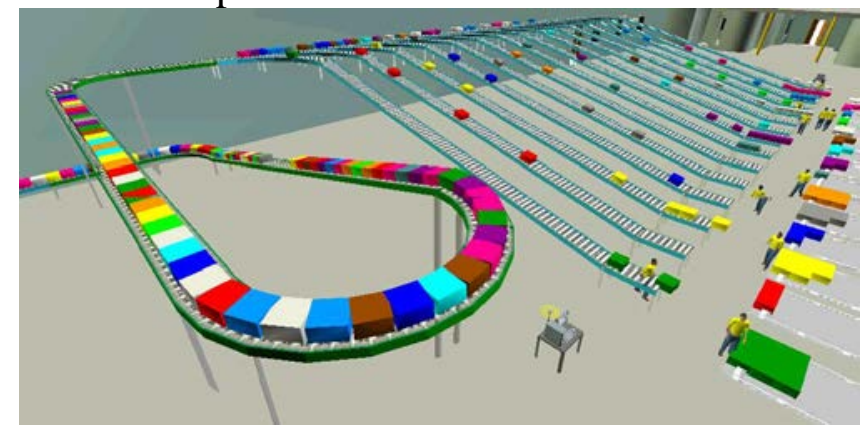

Figure 3 Power enterprise logistics model

\subsection{Information Scheduling}

In the work of electric power enterprise, the real-time data is very important, the enterprise can use the information obtained by the feedback as the reference basis for the stable operation of the power grid, and then define the many subsystems in the previous work, after the definition can find the amount, the actual situation of each department work, and the characteristics of the data operation, in order to do the cost control work well, it is necessary for each department to do the 
public data collation work well, it should also be statistical data, and strengthen the consolidation, after the analysis, it can get the result of the feedback, the data mining structure, the data source, monitor, the data warehouse, the customer application is the unique part of the structure, the data processing work can be carried out smoothly, the data source is the bottom database; the data monitor can perceive the data of the source command, and then extract the data integration according to the data base.

\section{Conclusion}

With the development of information technology, the number of people using the Internet is increasing, and the data is growing at an amazing speed. During the period of data gradually expanding, it is difficult for the existing data processing tools to deal with the large volume of data group effectively, the database and data mining technology appear, solve the problem of large data volume, and strengthen the function, apply the database and data mining technology to the power industry, which can make the power industry develop towards the direction of intelligence and automation.

\section{References}

[1] Liu, Chao. data warehouse and the application of data mining technology in decision support systems. Journal of Tianjin Vocational College, vol. 21, no. 02, pp. 102-108, 2019.

[2] Cong, Monday. data mining technology and its application in power system load forecasting. Heilongjiang Science and Technology Information, no. 31, pp. 20, 2016.

[3] Shu, Sheng., Li, Zuxian. Application of data warehouse and data mining technology in army food procurement system and its value evaluation. Modern economic information, no. 02, pp. 379, 2015.

[4] Sun, Weixing. data mining technology and its application in power system load forecasting. New Technologies and Products in China, no. 23, pp. 7, 2014.

[5] Chen, Dong. Research on the application of data warehouse and data mining technology in securities customer relationship management system. Digital Technology and Applications, no. 08, pp. 66+68, 2013. 